\title{
Relazioni pericolose. I gesuati e la povertà
}

\author{
Michele Lodone
}

\begin{abstract}
SommaRıo: Alle origini, la «brigata» dei gesuati presentava alcuni tratti di non conformismo religioso: la vita itinerante, le vesti rappezzate, il legame carismatico (non istituzionale) che univa i «povari di Cristo». Questi elementi fecero sì che, agli occhi dell'autorità ecclesiastica, Giovanni Colombini e i suoi compagni apparissero simili ai gruppi dissidenti allora presenti in Italia centrale, come i fraticelli. Alla luce di tale prossimità - apparente, ma rischiosa - il saggio si concentra su tre momenti che contribuirono a definire l'identità stessa dei gesuati: l'inquisitio cui nel 1367, a Viterbo, furono sottoposti Colombini e la «brigata de' povari»; il riemergere di dubbi e sospetti a Siena (1412), Bologna (1425) e Venezia (1436-1437); la metamorfosi dell'ideale di povertà, da concreta proposta di vita a irripetibile paradigma spirituale.
\end{abstract}

Nel primo pomeriggio del 15 settembre 1388, un mercoledì, la piazza principale di Perugia (l'attuale piazza IV Novembre, tra il palazzo dei Priori e la cattedrale di San Lorenzo), fu teatro di un evento singolare. O, quantomeno, abbastanza clamoroso da attirare l'attenzione di un cronista cittadino, che così lo decrisse pochi giorni dopo:

A queste dì passate, che fu adì 15 de setembre, in mercoredì, a ora de nona, lì in piaza uno fraticello de quelli Jesuati subito in uno stante gettò via el suo mantello et il capuccio, et levò grandissimi strida, et fugì via per la piazza sempre gridando. Et non fu mai più veduto, et non se sa quello che se volesse dire ${ }^{1}$.

Il significato della performance, per noi poco chiaro, sfuggiva a quanto pare anche ai contemporanei. Alla scarsa trasparenza dei gesti - spogliarsi, gridare e fuggire gridando - si sovrapponeva del resto un'opacità ulteriore, che riguardava l'identità stessa del loro attore. «Uno fraticello de quelli Jesuati»: l'accostamento dei due termini, sorto forse in modo spontaneo nella mente dell'osservatore (e in tal caso tanto più significativo), non era casuale. La «brigata $\gg$ dei gesuati, nata poco più di vent'anni prima dall'esperienza religiosa

1 Cronaca della città di Perugia dal 1309 al 1491 nota col nome di "Diario del Graziani", in F. Bonaini, A. Fabretti, F.L. Polidori (a cura di), Cronache e storie inedite della città di Perugia dal MCL al MDLXIII, «Archivio storico italiano», XVI (1), 1850, pp. 71-750: 232 (ho leggermente modificato la punteggiatura).

Michele Lodone, University of Venice Ca' Foscari, Italy, michele.lodone@unive.it, 0000-0002-1014-5532 FUP Best Practice in Scholarly Publishing (DOI 10.36253/fup_best_practice)

Michele Lodone, Relazioni pericolose. I gesuati e la povertà, pp. 177-187, (c) 2020 Author(s), CC BY 4.0 International, DOI 10.36253/978-88-5518-228-7.14, in Isabella Gagliardi (edited by), Le vestigia dei gesuati. L'eredità culturale del Colombini e dei suoi seguaci, () 2020 Author(s), content CC BY 4.0 International, metadata CCO 1.0 Universal, published by Firenze University Press (www.fupress.com), ISBN 978-88-5518-228-7 (PDF), DOI 10.36253/978-88-5518-228-7 
del mercante senese Giovanni Colombini, fin dalle origini aveva dovuto rendere conto delle proprie somiglianze di famiglia con alcuni aspetti del variegato universo del non conformismo religioso. Il fondamento carismatico, non istituzionale, che univa $\mathrm{i}$ «povari di Cristo»; la povertà cui il loro nome stesso rimandava, e che inizialmente si manifestava nella scelta di vita itinerante $\mathrm{e}$ nelle vesti rappezzate: questi elementi avevano reso Colombini e i suoi compagni, agli occhi dell'autorità ecclesiastica, pericolosamente simili alla tradizione francescana dissidente dei fraticelli. Tale congenita prossimità - più apparente che profonda, ma nondimeno rischiosa - contribuì fortemente, almeno nel primo secolo di storia dei gesuati, a definire la loro stessa identità. Un'identità che intendeva porsi anzitutto sul piano dell'esperienza religiosa, ma che non poté evitare, per sopravvivere, di passare anche attraverso un processo di inquadramento istituzionale, imposto o negoziato.

Nelle pagine che seguono, si prenderanno in considerazione tre momenti che scandiscono la storia dell'identità gesuata, nella sua complessa interazione con la povertà (pensata o vissuta), e nei suoi tentativi di prendere le distanze da realtà e immagini scomode, se non moleste, per la loro vicinanza. L'inquisitio cui nel 1367, a Viterbo, furono sottoposti Giovanni Colombini e la sua «brigata de' povari» rappresentò il primo e decisivo spartiacque: in quel frangente, sulle ragioni storiche che portarono alcuni esponenti della curia ad associare la neonata proposta religiosa a quella dei fraticelli, ebbero la meglio le oggettive differenze tra il dissenso di questi ultimi e l'atteggiamento umile e obbediente di Colombini e compagni (differenze che le lettere di Giovanni dalle Celle permettono di cogliere da una prospettiva singolare e particolarmente acuta).

L'approvazione pontificia - del resto solo orale - non bastò, tuttavia, a mettere la brigata definitivamente al riparo. Di qui il permanere di dubbi e sospetti, nella prima metà del Quattrocento, e il loro riemergere a Siena nel 1412, a Bologna nel 1425, a Venezia nel 1436-1437. Di fronte a tali emergenze, la nuova congregazione dimostrò un' insospettata capacità di tutelarsi, anche e soprattutto sul piano giuridico e normativo. Ma il significato stesso della povertà, nel passaggio dell'esperienza gesuata dal carisma all'istituzione o più precisamente, in tre tempi, dalla brigata alla congregazione all'Ordine a tutti gli effetti - mutò inevitabilmente. Perché negli ultimi decenni di esistenza dei gesuati la scelta della povertà, con i suoi annessi e connessi dell'itineranza, della vita eremitica e del lavoro manuale, subì una metamorfosi tale da trasformare una concreta proposta di vita in un paradigma spirituale assoluto e irripetibile.

1. «Se la povertà è qui a sospetto, questo non è colpa di coloro che reggono la Chiesa». La brigata de' povari, l'inquisitio di Viterbo (1367) e il fantasma dei fraticelli

«Gridiamo el dì e la notte, per le vie e per le piazze, el nome de Cristo benedetto»: testimonianze simili sono frequenti, nelle lettere o nelle biografie di 
Giovanni Colombini ${ }^{2}$. Tra questi episodi di gioiosa (e rumorosa) 'santa pazzia', ha un'importanza e un significato particolare quello verificatosi a Corneto, il 4 giugno 1367. Quel giorno Colombini e i suoi compagni, «tutti cogli ulivi in capo e in mano, con gridare sempre: "laldato Cristo", e "viva il Santo Padre"», si presentarono allo sbarco del pontefice Urbano V, in viaggio da Avignone a Roma; «e tutto el campo - scrisse lo stesso Colombini - con lalde e con cose mettemmo a rotta e a festa» ${ }^{3}$.

Il resoconto dell'ormai ex mercante senese, affidato a una serie di lettere inviate in quei giorni alle monache di Santa Bonda, al contempo madri e figlie spirituali, permette di seguire gli eventi successivi quasi in presa diretta (e tale resoconto è alla base della successiva storiografia gesuata: da Feo Belcari e Antonio Bettini, sui quali torneremo tra breve, fino a Paolo Morigia ${ }^{4}$ ). Il festoso scompiglio portato dai «poveregli» toscani, che si posero al seguito di Urbano $V$ nel tragitto da Corneto a Toscanella e quindi a Viterbo, suscitò, presso la corte papale, sentimenti ambivalenti. Alla benevolenza e curiosità di alcuni, tra i quali spicca il fratello del papa, il vescovo di Avignone e cardinale di San Pietro in Vincoli Anglic de Grimoard $^{5}$, corrispose la diffidenza, più o meno apertamente ostile, di altri.

Delle ragioni di tali sospetti, Colombini prese gradualmente coscienza: in modo all'inizio generico ( $\ll$ tante so' le eresia tra le genti, che non si può credere che noi siamo netti né puri per molti»), ma via via più nitido («per cierto tenete che chiunque è diventato povaro abbia presa opinione o 'resia, e subito ereticano contra la Chiesa, unde noi, non essendo avisati, ci semo messi a grande rischio $\left.{ }^{6}\right)$. I sospetti, è chiaro, erano rivolti al pauperismo non disciplinato dei membri della brigata, alla loro povertà che era «in abominazione quasi al più delle genti», e in particolare ai prelati ${ }^{7}$. Questi sospetti colpirono negativamente gli entusiasti penitenti toscani. Alcuni abbandonarono la causa e tornarono alla $\ll$ miseria del mondo» ${ }^{8}$; ma Colombini e altri suoi compagni scelsero la via della pazienza e

Vd. G. Colombini, Lettere, a cura di A. Bartoli, Balatresi, Lucca 1856, p. 56.

3 Ivi, p. 218. Su Urbano V (1362-1370), la sua politica di riforma del clero e i suoi tentativi di arginare le esperienze di non conformismo religioso vd., in breve, la voce di M. Hayez, in Enciclopedia dei Papi, II, Istituto della Enciclopedia Italiana, Roma 2000, pp. 542-550.

4 Vd. F. Belcari, La vita del beato Giovanni Colombini da Siena, fondatore dell'Ordine dei poveri Iesuati, e de' suoi compagni, in Venetia, nella contra' di S. Maria Formosa, 1554, cc.n.n., capitoli LVIII-LX; Antonio Bettini, De statu Iesuatorum, in Città del Vaticano, Bibloteca Apostolica Vaticana, Chig. i.vi.227, c. 11r (cit. da I. Gagliardi, Li Trofei della Croce. L'esperienza gesuata e la società lucchese tra Medio Evo ed Età Moderna, Edizioni di Storia e Letteratura, Roma 2005, p. 14); Paolo Morigia, Paradiso de' Giesuati, nel quale si racconta l'origine dell'Ordine de' Giesuati di S. Girolamo et la vita del b. Giovanni Colombini, fondatore di esso Ordine, con parte delle sante vite d'alcuni de' suoi discepoli et imitatori, in Vinetia, presso Domenico e Gio. Battista Guerra fratelli, 1582, pp. 69-71.

5 Su cuivd. il profilo di A. Gamberini, Grimoard, Anglic de, in Dizionario Biografico degli Italiani [d'ora in poi DBI], LIX, Istituto dell'Enciclopedia Italiana, Roma 2002, pp. 679-683.

6 Giovanni Colombini, Lettere, cit., pp. 218, 229.

7 Ivi, pp. 236.

8 Ivi, pp. 224. 
dell'umiltà, mantenendo un atteggiamento di assoluta obbedienza nei confronti della gerarchia ecclesiastica e anche di ammirazione per i cardinali della curia avignonese. Questi «hanno più umiltà», scriveva Colombini, «negli stati grandi e nelle molte ricchezze, che noi povari superbi nello stato vile ed abbietto»; «e per certo tenete», aggiungeva di seguito, «che se la povertà è qui a sospetto, questo non è colpa di coloro che reggono la Chiesa, ma de' povari superbi ed erranti»?

La paziente e umile obbedienza di Colombini fu molto apprezzata. Il pontefice in persona donò ai penitenti delle vesti bianche, che servivano a distinguere anche esteriormente i membri della compagnia, fino ad allora vestiti con abiti dimessi e rappezzati, da quelli di altri movimenti pauperistici eterodossi. Colombini si rallegrò del dono, e del fatto che a Viterbo si cominciasse a chiamare lui e i suoi compagni «e povari del Papa $»^{10}$. La brigata fu sottoposta a un esame inquisitoriale, volto a verificarne l'ortodossia: ma le risposte di Giovanni Colombini e di Francesco di Mino Vincenti, già postisi sotto la tutela e la guida del cardinal de Grimoard, furono giudicate soddisfacenti dall'inquisitore domenicano Guillaume Sudre (de La Sudrie), già vescovo di Marsiglia e pochi mesi prima nominato cardinale dei Santi Giovanni e Paolo ${ }^{11}$.

La condotta prudente di Colombini fece sì che la forma di vita dei «poveregli» - i cui membri presero da allora il nome di gesuati - fosse approvata dal pontefice (per quanto solo oralmente, ovvero vivae vocis oraculo). Nondimeno, il cenno di Colombini al «grande rischio» in cui si era messo, insieme ai suoi compagni, non è affatto esagerato. Il rigoroso pauperismo, l'itineranza, la natura spontanea, istituzionalmente non inquadrata (e, come mostra la storia stessa dei gesuati, non facilmente inquadrabile) erano elementi che rendevano la brigata inquietante, agli occhi della gerarchia ecclesiastica del tempo. Non solo, genericamente, per la lunga storia dell'associazione tra povertà ed eresia ${ }^{12}$, ma anche per l'attualità scottante che allora, alla questione della povertà, conferiva il vivace dissenso religioso dei fraticelli. Con tale diminutivo, cui rimaneva un' insopprimibile polivalenza semantica, le fonti del tempo facevano spesso riferimento al mondo del dissenso francescano: un movimento religioso complesso e tutt'altro che unitario fin dalle sue origini, che rimandano sia agli scontri sull'osservanza della Regola francescana tra i cosiddetti "spirituali" e la comunità dell'Ordine dei frati Minori, sia al successivo conflitto tra la dirigenza stessa dell'Ordine, guidata dal ministro generale Michele da Cesena, e papa Giovanni XXII sul tema della povertà evangelica ${ }^{13}$.

9 Ivi, pp. 228-229.

10 Ivi, p. 228.

11 Sul dotto teologo domenicano, molto vicino al pontefice, vd. R. Fage, Guillaume Sudre, cardinal limousin, Roche, Brive 1880; S.L. Forte, The Cardinal-Protector of the Dominican Order, Angelicum, Roma 1959, pp. 9-14.

12 Vd. L. Paolini, Esiti ereticali della conversione alla povertà, in E. Menestò (a cura di), La conversione alla povertà nell'Italia dei secoli XII-XIV, CISAM, Spoleto 1991, pp. 127-186.

$13 \mathrm{Vd}$. S. Piron, Le mouvement clandestin des dissidents franciscains au milieu du XIV siècle, «Oliviana», III, 2009, <http://oliviana.revues.org/337?\&id=337> (10/2020). 
Nelle sue lettere, Colombini non parla esplicitamente di fraticelli, ma di opinione eretica in materia di povertà. Il riferimento, tuttavia, sembra inequivocabile: intorno alla metà del Trecento la formula «opinione eretica» si era già cristallizzata (in riferimento alla povertà di Cristo e degli apostoli e all'eresia di Giovanni XXII, che l'aveva negata, nonché dei pontefici suoi successori). All'ombra dei fraticelli sull'incontro di Viterbo, nel secolo seguente, accennarono poi esplicitamente Feo Belcari e Antonio Bettini nei loro già menzionati resoconti delle origini gesuate. Il contesto di composizione delle due opere contribuisce a spiegare il fatto. La Vita di Belcari risale al 1449, anno in cui a Fabriano, davanti al papa Niccolò $V$, furono condannati al rogo numerosi fraticelli ${ }^{14}$. Nel 1450, proprio a Firenze (dove Belcari viveva), l'arcivescovo Antonino fece giustiziare come eretico impenitente, incantatore e seguace dell'eresia dei fraticelli il medico di Montecatini Giovanni Cani ${ }^{15}$. Bettini, dal canto suo, scriveva negli anni '60 del Quattrocento, e appunto tra il 1455 e il 1466-1467 ebbero luogo altri processi contro i fraticelli (l'ultimo dei quali indetto a Roma, con grande dispiegamento di apparati pubblici e di confutazioni teologiche, da Paolo II $)^{16}$.

Detto ciò, è molto probabile che lo spettro ereticale cui Colombini stesso, un secolo prima, faceva riferimento fosse proprio quello dei fraticelli, alla luce sia della loro vivace presenza, negli anni dell'inquisitio viterbese, tra Toscana, Lazio, Umbria e Marche; sia del fondamento canonico della condanna dei fraticelli da parte dell'autorità ecclesiastica - ovvero la bolla Sancta Romana Ecclesia, promulgata nel 1317 da Giovanni XXII ${ }^{17}$.

Dal primo punto di vista, l'attualità del dissenso dei fraticelli, poco dopo la metà del Trecento, è testimoniata dal ripetersi delle azioni repressive nei loro confronti. Ai tentativi del cardinale Gil (Egidio) de Albornoz, era seguita infatti l'azione del vescovo di Narni e inquisitore per la Tuscia frate Guglielmo, incaricato dallo stesso Urbano V. Nel settembre del 1368 (un anno e pochi mesi dopo l'incontro del papa con la brigata di Giovanni Colombini), frate Guglielmo di Narni condannò al rogo alcuni fraticelli a Roma e, forse, proprio a Viterbo ${ }^{18}$.

14 Vd. S. Simoncini, «Et inventos convincere non vi $\gg:$ la repressione dei fraticelli «de opinione» tra Martino Ve Niccolò V, in E.M. Vecchi (a cura di), Papato, stati regionali e Lunigiana nell'età di Niccolò $V$, «Memorie della Accademia lunigianese di scienze "Giovanni Capellini"», LXXIII, 2004, pp. 85-109.

15 Vd.P. Howard, "It is a great disgrace for our city": Archbishop Antoninus and Heresy in Renaissance Florence, in J. Spinks, D. Eichberger (eds.), Religion, the Supernatural and Visual Culture in Early Modern Europe. An album amicorum for Charles Zika, Brill, Leiden 2015, pp. 103-125.

16 Vd. J. Monfasani, The Fraticelli and Clerical Wealth in Quattrocento Rome, in J. Monfasani, R.G. Musto (eds.), Renaissance Society and Culture: Essays in Honour of E.F. Rice jr., Italica Press, New York 1991, pp. 177-195.

17 Edizione critica in J. Tarrant (a cura di), Extravagantes Iohannis XXII, Biblioteca Apostolica Vaticana, Città del Vaticano 1983, pp. 198-204.

18 Le fonti riguardanti questo episodio, in più punti contraddittorie, sono analizzate da F.A. Angeli, Fonti e problemi riguardanti la storia del fraticellismo nel Lazio (secc. XIV-XV), tesi di laurea, Università degli Studi “La Sapienza” di Roma, relatrice S. Di Mattia Spirito, a.a. 1992-1993, pp. 136-143. 
Quanto alla Sancta Romana, con essa il papa caorsino aveva condannato per la prima volta $\mathrm{i}$ «fraticelli seu fratres de paupere vita», insieme ad altri raggruppamenti penitenziali e beghinali, contestando loro di non aver ricevuto la necessaria approvazione, e di diffondere non specificate dottrine ereticali ${ }^{19}$. Erano condizioni che si potevano facilmente sovrapporre a quelle in cui si trovavano Colombini e i suoi compagni. Non è un caso che questi ultimi, nella nota di spese del papa per l'acquisto delle già menzionate vesti bianche, fossero chiamati proprio fratiselli: un termine la cui valenza generica, di vicinanza ad esperienze eremitiche e pauperistiche, dopo la bolla di Giovanni XXII, conviveva sempre più spesso con un'accezione ereticale ${ }^{20}$. La linea di confine con l'eterodossia era resa potenzialmente ancora più sottile da altre relazioni pericolose intrattenute dalla brigata dei poveri: mentre questi si trovavano a Viterbo, infatti, un ignoto maestro senese aveva denunciato al papa come sospetta la loro stretta relazione con le monache di Santa Bonda ${ }^{21}$.

Se non fosse stato per l'atteggiamento di totale obbedienza e per la prudente capacità di autotutelarsi di Giovanni Colombini e di Francesco di Mino Vincenti, queste somiglianze di famiglia avrebbero potuto costare caro. Certo, ai nostri occhi, le origini dell'esperienza di Giovanni, di Francesco e dei loro compagni rimandano a un contesto diverso da quelli in cui si sviluppò il dissenso religioso dei fraticelli: a un contesto senese monastico (per gli stretti rapporti col certosino Pietro Petroni) e confraternale (per l'appartenenza alla compagnia di Santa Maria della Scala), i cui molteplici apporti furono fusi in un'esperienza religiosa ed esistenziale sicuramente originale ${ }^{22}$. Ma la confusione terminologica el'opacità delle distinzioni identitarie, allora, rappresentavano un elemento di rischio tutt'altro che trascurabile.

A rifuggire dal senno di poi, in modo da cogliere con gli occhi di un contemporaneo le differenze tra le 'proposte cristiane' dei fraticelli e dei futuri gesuati, può aiutare l'epistolario di Giovanni dalle Celle. Il dotto monaco vallombrosano scrisse - a distanza di un decennio - sia ai «poveri novelli di Cristo» (tra il 1363 e il 1368), sia ai «fraticelli della povera vita» e ai loro seguaci o simpatizzanti

19 Vd. R. Lambertini, Spirituali e fraticelli: le molte anime della dissidenza francescana nelle Marche tra XIII e XV secolo, in L. Pellegrini, R. Paciocco (a cura di), I francescani nelle Marche. Secoli XIII-XVI, Silvana, Cinisello Balsamo 2000, pp. 38-53: 45.

20 Sulla complessa e a tratti sfuggente storia del termine, vd. G. Tognetti, I fraticelli, il principio di povertà ed i secolari, «Bullettino dell'Istituto storico italiano per il Medio Evo e Archivio muratoriano», XC, 1982-1983, pp. 77-145; R. Lambertini, «Non so che fraticelli...»: identità e tensioni minoritiche nella Marchia di Angelo Clareno, nell'opera collettiva Angelo Clareno francescano, CISAM, Spoleto 2007, pp. 229-261.

21 Vd. I. Gagliardi, I "Pauperes Yesuati" tra esperienze religiose e conflitti istituzionali, Herder, Roma 2004, p. 270. Sulla questione vd., più in generale, A. Benvenuti Papi, Le donne di Giovanni Colombini. «Parmi mill'anni di vederti povera e ribalda», in Ead. «In castro poenitentiae». Santità e società femminile nell'Italia medievale, Herder, Roma 1990, pp. 415-528.

22 I. Gagliardi, «Novellus pazzus». Storie di santi medievali tra il Mar Caspio e il Mar Mediterreaneo (secc. IV-XIV), Società Editrice Fiorentina, Firenze 2017, pp. 173-176. 
laici (tra il 1378 e il 1381) ${ }^{23}$. Nelle sue lettere spicca il contrasto tra l'approvazione dell'umile obbedienza dei primi, e la riprovazione della superbia scismatica dei secondi; tra la polemica contro le pretese ecclesiologiche totalizzanti della povertà dei primi, e l'apprezzamento, per converso, dell'ispirazione monastica cui Giovanni riconduceva la povertà dei secondi. Non è detto che, in tal modo, l'eremita di Vallombrosa cogliesse a pieno l'originalità dell'esperienza cristiana di Colombini e della sua brigata; ma sicuramente egli sottolineava un aspetto fondamentale della loro vocazione, votata alla povertà come forma di libertà da ogni sollecitudine mondana, di «libertà per Cristo $»^{24}$. Una povertà molto diversa da quella polemicamente rivendicata, negli stessi anni, dai fraticelli.

2. «Res est nova, et per consequens prohibita». I sospetti sull'ortodossia della congregazione (prima metà del XV secolo)

Nel 1367 la brigata dei poveri, da allora noti come gesuati, ottenne il riconoscimento della propria ortodossia da parte del papa, e modificò la propria natura spontanea per trasformarsi, seppure solo in parte e con un processo che sarebbe durato vari decenni, in una congregazione religiosa. Non fu semplice per Colombini e compagni mantenere ferma la propria vocazione, che rischiava di essere snaturata ${ }^{25}$. Il passaggio, del resto, fu tutt'altro che indolore. Lo testimoniano i cenni delle fonti alle defezioni di vari membri della brigata, e un episodio - per noi completamente oscuro - che vide un certo Biliotto abbandonare il gruppo, diffamarlo di fronte alla Curia e morire impiccato ad Arezzo con quattordici compagni ${ }^{26}$. Non si trattò dell'ultima volta in cui le autorità ecclesiastiche guardarono ai gesuati con una certa diffidenza. Tre episodi, infatti, attestano il permanere o riemergere di sospetti di eterodossia nella prima metà del Quattrocento.

Il primo si verificò a Siena, dove nel 1412 il vescovo Antonio Casini informò il priore allora in carica, Andrea di Ambrogio, che la compagnia di Santa Maria della Scala accoglieva dei fraticelli dell'opinione, tra i quali era il cappellano Giacomo da Lucca. Non sappiamo molto, su quanto ne seguì: dalle fonti interne alla confraternite risulta che i suoi membri provvidero a espellere i sospetti,

23 G. dalle Celle, L. Marsili, Lettere, a cura di F. Giambonini, Olschki, Firenze 1991, II, pp. 331-349 ai gesuati; pp. 379-468, 499-515 ai fraticelli (per la datazione, I, pp. 184-185, 188-193). Vd. rispettivamente Gagliardi, I "Pauperes Yesuati”, cit., pp. 252-254, 310-312; C. Gennaro, Giovanni dalle Celle e i fraticelli, «Rivista di storia e letteratura religiosa», XXXV, 1999, pp. 31-69.

24 Vd. D. Rando, Le avventure della «devotio» nell'Italia del Tre-Quattrocento fra storia e storiografia, in M. Derwich e M. Staub (eds.), Die 'Neue Frömmigkeit' in Europa im Spätmittelalter, Vandenhoeck and Ruprecht, Göttingen 2004, pp. 331-351: 346.

25 Vd. C. Gennaro, Giovanni Colombini e la sua «brigata», «Bullettino dell'Istituto storico italiano per il Medio Evo e Archivio muratoriano», LXXXI, 1969, pp. 237-271: 264.

26 Vd. F. Belcari, La vita del beato Giovanni Colombini, cc. n.n., capitoli XXVI e LVII; Morigia, Paradiso de' Giesuati, cit., pp. 70-71. 
e che sospesero per un periodo precauzionale Giacomo da Lucca (prima di riammetterlo nel gennaio del 1414) ${ }^{27}$.

La nebulosità istituzionale del movimento gesuato provocò poi, negli anni seguenti, due vere e proprie inquisitiones. La prima ebbe luogo a Bologna, nel 1425 o poco prima, in seguito a una denuncia esposta dal clero locale al vescovo Niccolò Albergati ${ }^{28}$. Le suspiciones nutrite nei confronti dei gesuati, sulla scia della Sancta romana, si appuntarono in particolare sulla loro novità ( $\ll$ res est nova, et per consequens prohibita»), e sul fatto che dei meri layci pronunciassero i voti di povertà, castità e obbedienza senza un adeguato riconoscimento da parte dell'autorità ecclesiastica. Il processo bolognese segna una svolta importante, nella storia della congregazione gesuata. In questa occasione, i suoi membri si munirono anzitutto di un consilium giuridico fondato sulle dichiarazioni di giganti del foro come Francesco Zabarella e Paolo de Leazariis, che scagionarono autorevolmente ifratres dall'accusa di contravvenire alla Sancta Romana ${ }^{29}$. Inoltre, essi promulgarono per la prima volta delle Constitutiones, approvate ufficialmente nell'aprile del 1426 da Albergati stesso e destinate ad aprire un «periodo di razionalizzazione interna e di attività normalizzante» ${ }^{30}$.

La seconda inquisitio quattrocentesca si svolse, invece, tra il 1436 e 1437 a Venezia, dove Eugenio IV assegnò l'indagine a due uomini di fiducia, che esaminassero la questione tanto sul piano teologico e devozionale quanto sul piano giuridico: il vescovo di Castello Lorenzo Giustiniani e il frate Minore osservante Giovanni da Capistrano ${ }^{31}$. Questa volta lo spauracchio ereticale era rappresentato dalla fantomatica setta del Libero Spirito, cui alcuni gesuati veneziani erano sospettati di avere aderito. L'ombra dei fraticelli, tuttavia, restava sullo sfondo, dal momento che ancora una volta i gesuati si trovarono a dover mettere a tacere l'accusa ricorrente di non aver ottemperato alle direttive della Sancta Romana in materia di approvazione di nuovi gruppi religiosi promossi da laici. In ogni caso,

27 Vd. Gagliardi, I "Pauperes Yesuati”, cit., pp. 70-71.

28 Sul disegno riformatore di Albergati e sulla sua opera pastorale vd. R. Parmeggiani, Visite pastorali e riforma a Bologna durante l'episcopato di Niccolò Albergati (1417-1443), «Rivista di storia della Chiesa in Italia», LXIX, 2015, pp. 21-47.

29 Il Consilium - che sottolinea peraltro la legittimità della questua dei gesuati, contrapposta ai benefici goduti da prelati indegni - si legge in G. Dufner, Geschichte der Jesuaten, Edizioni di Storia e Letteratura, Roma 1975, pp. 90-97.

30 Vd. Gagliardi, I "Pauperes Yesuati", cit., pp. 306-312.

31 Su Giustiniani, nominato vescovo di Castello da Eugenio stesso nel 1433, vd. la voce di G. Del Torre, in DBI, LXVI, 2006, pp. 73-77. Su Giovanni da Capestrano e la sua azione inquisitoriale, si rimanda invece alla voce di L. Pellegrini, in A. Prosperi (a cura di), Dizionario storico dell'Inquisizione, con la collaborazione di V. Lavenia e J. Tedeschi, Edizioni della Normale, Pisa 2010, II, pp. 702-703; e per i suoi stretti rapporti con Eugenio IV, che attendono ancora una ricostruzione compiuta, vd. F. Sedda, «Corpus Epistolarum Capistrani» (CEC): An Overview of the Database of John of Capistrano's Epistolary, in P. Kras, H. Manikowska, M. Starzyński, A. Zajchowska-Bołtromiuk (eds.), Corpus epistolarum Ioannis de Capistrano, I: Epistolae annis MCDLI-MCDLVI scriptae quae ad res gestas Poloniae et Silesiae spectant, Polish Academy of Sciences - Wydawnictwo KUL, Warszawa-Lublin 2018, pp. 35-46: 45. 
i gesuati veneti furono giudicati irreprensibili dai loro inquisitori, forse anche grazie alla conoscenza diretta che di loro aveva il papa stesso, il veneziano Eugenio IV. Il quale del resto, durante il suo pontificato, fornì dei privilegi anche ad altri gruppi religiosi di confine, per lo più formati da terziari e terziarie, affinché si mettessero al riparo dall'onda lunga delle prescrizioni della Sancta Romana ${ }^{32}$.

3. Il paradiso delle origini. La povertà dei gesuati da proposta di vita a paradigma spirituale

A partire dalla seconda metà del Quattrocento, i sospetti di eterodossia suscitati dal pauperismo dei gesuati persero il significato che potevano avere nel primo secolo di storia della brigata (poi divenuta congregazione). In quei decenni, gli ideali stessi di povertà cristiana andavano perdendo il ruolo teologico $\mathrm{e}$ politico-ecclesiologico centrale rivestito fino ad allora ${ }^{33}$. Ma questo mutamento di prospettive può consentire di riflettere anche, da una prospettiva particolare, sulla più ampia metamorfosi dell'identità gesuata.

Dalle grandi discussioni sulla povertà e la ricchezza della Chiesa i gesuati si erano sempre tenuti alla larga, puntando sul lavoro manuale (la lavorazione del vetro, la distillazione di acque aromatiche e medicinali, la copiatura di manoscritti) ${ }^{34}$. Essi seguirono la loro vocazione sociale, anteponendo la carità alla perfezione individuale, e coltivarono le arti meccaniche; il che implicò il definitivo abbandono (o meglio, l'interiorizzazione), a partire dalla metà del Quattrocento, della vita eremitica che aveva fatto parte della loro esperienza delle origini, e la neutralizzazione della sua potenziale carica dissidente ${ }^{35}$.

Questa esperienza era nata dal recupero e dalla riattivazione, da parte di Giovanni Colombini e dei suoi compagni, di modelli di santità precedente: Maria Egiziaca anzitutto, la cui storia - letta da Giovanni su invito della moglie Biagia, per ingannare il tempo nell'attesa del pranzo - lo spinse a quanto pare alla conversione e alla rinuncia al mondo; ma anche Alessio, Francesco d'Assisi e altri ancora $^{36}$. Quando Colombini stesso, tra la fine del Trecento e la seconda metà

32 Vd. Gagliardi, I "Pauperes Yesuati”, cit., pp. 325-337. Sulla politica di regolarizzazione promossa da Eugenio IV nei confronti dei gruppi di laici o terziari che rischiavano di incappare nelle censure della Sancta Romana Ecclesia, vd. A. Benvenuti Papi, Forme comunitarie (1982), in Ead., «In castro poenitentiae», cit., pp. 531-592: 536, 542 e passim.

33 L'argomento attende ancora uno studio adeguato; per il momento, vd. M. Lodone, La doppia povertà. Una inedita disputatio tra Giovanni XXII eMichele da Cesena, «Picenum Seraphicum», XXXI, 2017, pp. 91-116: 92-96.

34 Vd. I. Gagliardi, «Servono Dio con le sue mani». Le officine gesuate come segno di vita apostolica nel Tardo Medioevo e nella prima Età Moderna, «Ricerche di storia sociale e religiosa», XXXIX, 2010, pp. 65-85.

35 Vd. Ead., L'eremo nell'anima. I gesuati nel Quattrocento, in A. Vauchez (a cura di), Ermites de France et d'Italie ( $X I^{e}-X V^{e}$ siècle), École française de Rome, Roma 2003, pp. 429-459: 459; Rando, Le avventure della «devotio», cit., pp. 342-347.

36 Vd. Gagliardi, «Novellus pazzus», cit., pp. 170-178. 
del secolo seguente, divenne a sua volta un modello di santità, il contesto storico che aveva reso possibile e unica la sua esperienza cristiana era ormai lontano. Sempre più lontano, fino all'estremo tentativo di recupero da parte di Paolo Morigia, alla fine del Cinquecento ${ }^{37}$. Ma per Morigia - morto nel 1604, ultimo dei gesuati ad opporsi all'ormai imminente sacerdotalizzazione dell'Ordine (1606), e alla sua ormai prossima fine (1668) - quello di Giovanni Colombini e della sua brigata dei poveri era un paradigma di santità, più che di vita. Un paradigma per molti versi affascinante e venerabile, ma ormai difficilmente imitabile.

\section{Bibliografia}

Angeli F.A., Fonti e problemi riguardanti la storia del fraticellismo nel Lazio (secc. XIV$X V)$, tesi di laurea, Università degli Studi “La Sapienza” di Roma, relatrice S. Di Mattia Spirito, a.a. 1992-1993.

Belcari F., La vita del beato Giovanni Colombini da Siena, fondatore dell'Ordine dei poveri Iesuati, e de' suoi compagni, in Venetia, nella contra' di S. Maria Formosa, 1554.

Benvenuti Papi A., Le donne di Giovanni Colombini. «Parmi mill'anni di vederti povera e ribalda», in Ead. «In castro poenitentiae». Santità e società femminile nell'Italia medievale, Herder, Roma 1990, pp. 415-528.

Celle G. (dalle), Marsili L., Lettere, a cura di F. Giambonini, Olschki, Firenze 1991.

Colombini G., Lettere, a cura di A. Bartoli, Balatresi, Lucca 1856.

Cronaca della città di Perugia dal 1309 al 1491 nota col nome di "Diario del Graziani", in F. Bonaini, A. Fabretti, F.L. Polidori (a cura di), Cronache e storie inedite della città di Perugia dal MCL al MDLXIII, «Archivio storico italiano», XVI (1), 1850, pp. 71-750.

Del Torre G., voce Lorenzo Giustiniani, in DBI, LXVI, 2006, pp. 73-77.

Dufner G., Geschichte der Jesuaten, Edizioni di Storia e Letteratura, Roma 1975.

Fage R., Guillaume Sudre, cardinal limousin, Roche, Brive 1880.

Forte S.L., The Cardinal-Prolector of the Dominican Order, Angelicum, Roma 1959.

Hayez M., Urbano V, in Enciclopedia dei Papi, II, Istituto della Enciclopedia Italiana, Roma 2000, pp. 542-550.

Howard P., "It is a great disgrace for our city": Archbishop Antoninus and Heresy in Renaissance Florence, in J. Spinks, D. Eichberger (eds.), Religion, the Supernatural and Visual Culture in Early Modern Europe. An album amicorum for Charles Zika, Brill, Leiden 2015, pp. 103-125.

Gagliardi I., L'eremo nell'anima. I gesuati nel Quattrocento, in A. Vauchez (a cura di), Ermites de France et d'Italie ( $X I^{e}-X V^{e}$ siècle), École française de Rome, Roma 2003, pp. 429-459.

—, I "Pauperes Yesuati" tra esperienze religiose e conflitti istituzionali, Herder, Roma 2004.

-, «Servono Dio con le sue mani». Le officine gesuate come segno di vita apostolica nel Tardo Medioevo e nella prima Età Moderna, «Ricerche di storia sociale e religiosa», XXXIX, 2010, pp. 65-85.

—, Morigia, Paolo, in DBI, LXXVI, 2012, pp. 843-845.

—, «Novellus pazzus». Storie di santi medievali tra il Mar Caspio e il Mar Mediterreaneo (secc. IV-XIV), Società Editrice Fiorentina, Firenze 2017. 
Gamberini A., Grimoard, Anglic de, in DBI, LIX, Istituto dell'Enciclopedia Italiana, Roma 2002, pp. 679-683.

Gennaro C., Giovanni Colombini e la sua «brigata», «Bullettino dell'Istituto storico italiano per il Medio Evo e Archivio muratoriano», LXXXI, 1969, pp. 237-271.

—, Giovanni dalle Celle e i fraticelli, «Rivista di storia e letteratura religiosa», XXXV, 1999, pp. 31-69.

Lambertini R., Spirituali e fraticelli: le molte anime della dissidenza francescana nelle Marche tra XIII e XV secolo, in L. Pellegrini, R. Paciocco (a cura di), I francescani nelle Marche. Secoli XIII-XVI, Silvana, Cinisello Balsamo 2000, pp. 38-53.

—, «Non so che fraticelli...»: identità e tensioni minoritiche nella Marchia di Angelo Clareno, in Angelo Clareno francescano, CISAM, Spoleto 2007, pp. 229-261.

Monfasani J., The Fraticelli and Clerical Wealth in Quattrocento Rome, in J. Monfasani, R.G. Musto (eds.), Renaissance Society and Culture: Essays in Honour of E.F. Rice jr., Italica Press, New York 1991, pp. 177-195.

Morigia P., Paradiso de' Giesuati, nel quale si racconta l'origine dell'Ordine de' Giesuati di S. Girolamo et la vita del b. Giovanni Colombini, fondatore di esso Ordine, con parte delle sante vite d'alcuni de' suoi discepoli et imitatori, in Vinetia, presso Domenico e Gio. Battista Guerra fratelli, 1582.

Lodone M., La doppia povertà. Una inedita disputatio tra Giovanni XXII e Michele da Cesena, «Picenum Seraphicum», XXXI, 2017, pp. 91-116.

Paolini L., Esiti ereticali della conversione alla povertà, in E. Menestò (a cura di), La conversione alla povertà nell'Italia dei secoli XII-XIV, CISAM, Spoleto 1991, pp. 127-186.

Parmeggiani R., Visite pastorali e riforma a Bologna durante l'episcopato di Niccolò Albergati (1417-1443), «Rivista di storia della Chiesa in Italia», LXIX, 2015, pp. 21-47.

Pellegrini L., voce Giovanni da Capestrano, in A. Prosperi (a cura di), Dizionario storico dell'Inquisizione, con la collaborazione di V. Lavenia e J. Tedeschi, Edizioni della Normale, Pisa 2010, II, pp. 702-703.

Piron S., Le mouvement clandestin des dissidents franciscains au milieu $d u X I V^{e}$ siècle, «Oliviana», III, 2009, <http://oliviana.revues.org/337?\&id=337> (10/2020).

Rando D., Le avventure della «devotio» nell'Italia del Tre-Quattrocento fra storia e storiografia, in M. Derwich e M. Staub (eds.), Die 'Neue Frömmigkeit' in Europa im Spätmittelalter, Vandenhoeck and Ruprecht, Göttingen 2004, pp. 331-351.

Sedda F., «Corpus Epistolarum Capistrani» (CEC): An Overview of the Database of John of Capistrano's Epistolary, in P. Kras, H. Manikowska, M. Starzyński, A. ZajchowskaBołtromiuk (eds.), Corpus epistolarum Ioannis de Capistrano, I: Epistolae annis MCDLI-MCDLVI scriptae quae ad res gestas Poloniae et Silesiae spectant, Polish Academy of Sciences - Wydawnictwo KUL, Warszawa-Lublin 2018, pp. 35-46.

Simoncini S., «Et inventos convincere non vi $\gg:$ la repressione dei fraticelli «de opinione $\gg$ tra Martino Ve Niccolò V, in E.M. Vecchi (a cura di), Papato, stati regionali e Lunigiana nell'età di Niccolò $V$, «Memorie della Accademia lunigianese di scienze "Giovanni Capellini”», LXXIII, 2004, pp. 85-109.

Tarrant J. (a cura di), Extravagantes Iohannis XXII, Biblioteca Apostolica Vaticana, Città del Vaticano 1983.

Tognetti G., I fraticelli, il principio di povertà ed i secolari, «Bullettino dell'Istituto storico italiano per il Medio Evo e Archivio muratoriano», XC, 1982-1983, pp. 77-145. 